\title{
TISSUE-ENGINEERED HUMAN ASTHMATIC BRONCHIAL EQUIVALENTS
}

\author{
Jean-Sébastien Paquette ${ }^{1}$, Véronique Moulin ${ }^{1}$, Pierrot Tremblay ${ }^{1}$, Vincent Bernier ${ }^{1}$, Michel Boutet ${ }^{2}$, Michel Laviolette ${ }^{2}$, \\ François A. Auger ${ }^{1}$, Louis-Philippe Boulet ${ }^{2}$ and Francine Goulet ${ }^{1 *}$ \\ 'Laboratoire de Génie Tissulaire/LOEX, Hôpital de l'Enfant-Jésus du CHA and \\ ${ }^{2}$ Unité de Recherche en Pneumologie, Hôpital Laval, Québec, Canada.
}

\begin{abstract}
The isolation of human bronchial epithelial (HBEC) and fibroblastic cells (HBFC) from biopsies of asthmatic and non-asthmatic volunteers provided unique cellular materials to be used for the production of bioengineered bronchial equivalents (BE) in vitro. The HBEC are grown on a mesenchymal layer seeded with HBFC and the BE can be maintained for at least 15 days in culture. Under the BE culture conditions established previously, HBEC undergo differentiation into ciliated and goblet cells, within a pseudostratified organization comparable to human bronchi. We published previously the results from histologic and functional analyses of such BE produced exclusively using non-asthmatic HBEC and HBFC. We report here the comparative analyses of $\mathrm{BE}$ produced with non-asthmatic and asthmatic living HBEC and $\mathrm{HBFC}$ (naBE and aBE, respectively). Our data indicated that all asthmatic HBEC populations grown on a mesenchymal layer, containing nonasthmatic HBFC, slowly reached a confluent state but then detached from the matrix upon culture time. These BE appear to be very good models to study the mechanisms involved in asthma in vitro.
\end{abstract}

Key words: human bronchial equivalents, asthma, bioengineering.

\author{
*Address for correspondence: \\ Francine Goulet \\ Tissue Engineering Laboratory \\ Hôpital de l'Enfant-Jésus \\ $1401,18^{\mathrm{e}}$ rue \\ Québec, (Québec), GIS 4L8, Canada.
}

Telephone Number: (418) 649-0252 ext. 4344

FAX Number: (418) 649-5969

E-mail: chgfgo@hermes.ulaval.ca

\section{Introduction}

In our modern society, thousands of people suffer from mild to severe asthma. This bronchial disorder is mostly associated with mucosal inflammation and airway hyperresponsiveness (Jeffery et al., 1989; Djukanovic et al., 1990; Boulet et al., 1993). Histologic analyses of bronchial biopsies taken on asthmatic patients report an apparent basement membrane thickening most likely caused by sub-epithelial fibrosis. It is postulated that fibroblasts, involved in collagen synthesis and remodelling, could be responsible for the acute fibrosis in response to cytokines secreted by inflammatory or epithelial cells (Brewster et al., 1990; Roche, 1991; Gauldie et al., 1992). Another major change in the structural properties of asthmatic bronchi is their partial or complete desquamation upon the evolution of the disease (Jeffery et al., 1989). Up to now, the alterations of the bronchial tissues of asthmatic subjects remain poorly understood and the various hypotheses raised on the putative mechanisms responsible for the maintenance and the progression of these changes have to be assessed.

Besides live animals (often rats and dogs) (DiCosmo et al., 1995; Chung, 1996; Widdicombe, 1996; Shichinoke et al., 1996), several research groups use animal bronchial tissues (Opazo-Saez and Pare, 1994; Baeza-Squiban et al., 1994; Davenport and Nettesheim, 1996) or cells grown in monolayers (De Jong et al., 1994; Gray et al., 1996) as experimental models, to study various aspects of asthma in vitro. To overcome interindividual variations among animals used as experimental models, the number of subjects needed to perform each study has to be quite considerable. Moreover, animal models are complex and it becomes sometimes difficult to control all physiologic parameters which may modulate the results of comparative studies between non-asthmatic and asthmatic groups. In addition, some limitations are associated with bronchial cell monolayers, particularly because heterotypic cellular interactions are difficult to reproduce under these culture conditions.

Over the last decade, bioengineering has enlarged the possibilities to develop tissue models (Langer and Vacanti, 1993) by combining the conventional cell culture approaches to a concept according to which most cells can adopt specific three-dimensional orientation and organization in an extracellular matrix, in response to proper stimuli induced mechanically in vitro. This concept has been confirmed in various bioengineered tissues produced in culture, notably in skin, blood vessels and ligaments (Bellows et al., 1982; Bouvard et al., 1992; Lopez-Valle et al., 1992; L'Heureux et al., 1993; Auger et al., 1995; 
Goulet et al., 2000; Chakir et al., 2001; Paquette et al., 2003).

Thus, we have used several populations of the human bronchial cells isolated in our laboratory (Goulet et al., 1996a) from bronchial biopsies of asthmatic and non-asthmatic subjects, in an attempt to produce three-dimensional bronchial equivalents (BE) in vitro. For the first time, a bilayered BE containing human bronchial epithelial (HBEC) and fibroblastic cells (HBFC) isolated from asthmatic biopsies was obtained and maintained in culture for at least 15 days. An asthmatic HBEC layer grown on a mesenchymal counterpart seeded with asthmatic $\mathrm{HBFC}$ formed a BE referred to as asthmatic BE (a/aBE). Similarly, the non-asthmatic BE (na/naBE) was produced by combining non-asthmatic HBEC and HBFC in their respective layers. The interesting results from comparative analyses of these BEs are described in the present report.

\section{Subjects}

Non-smoking asthmatic and normal subjects aged from 20 to 45 years, evaluated at the Laval Hospital asthma clinic, were enrolled in the study. The study was approved by the local Ethics Committee and subjects had given informed written consent. The normal subjects had a $\mathrm{PC}_{20}$ value (metacholine provocation, see below) over $16 \mathrm{mg} / \mathrm{ml}$. All asthmatic subjects had a diagnosis of asthma according to the American Thoracic Society criteria (American Thoracic Society, 1987). All were atopic with at least one positive response to common allergens on allergy skin prick tests. Their asthma required only an inhaled $\beta 2$ agonist agent on demand. None of the subjects reported a respiratory infection or an increase in asthma symptoms in the month preceding the study. They were not currently exposed to allergens to which they were sensitized apart from house-dust.

Spirometry and response to inhaled methacholine were measured according to standardized procedures using aerosols generated with a Wright's nebulizer at tidal breathing for periods of 2 minutes (output $=0.13 \mathrm{ml} / \mathrm{min}$ ) (Juniper et al., 1991). The provocative concentration of methacholine inducing a $20 \%$ fall in $\mathrm{FEV}_{1}$, the $\mathrm{PC}_{20}$, was determined. Skin prick tests were performed with a battery of common airborne allergens. Atopy was considered to be present if there was one or more positive response ( $\geq 3 \mathrm{~mm}$ wheal) to the inhalant allergens, with a positive reaction to histamine phosphate but not the diluent.

\section{Bronchoscopy and bronchial biopsies}

Before the bronchoscopy, a $200 \mu \mathrm{g}$ dose of salbutamol was given using a metered-dose inhaler. All subjects received oxygen at $51 / \mathrm{min}$ by nasal catheter during bronchoscopy. After local anaesthesia of the throat, larynx and bronchi with $2 \%$ and $4 \%$ xylocaine, the flexible bronchoscope (Olympus OES 10 fiberscope, Olympus, Markham, Canada) was introduced into the bronchial tree and ten bronchial biopsies were taken from the carinae of the right upper and middle lobes and the segmental bronchi of the upper and lower lobes on both sides using conventional forceps. Vital signs, electrocardiograph and oximetry were recorded throughout the procedure.

\section{Human bronchial cell isolation}

The human bronchial cells were isolated by a new enzymatic technique and amplified in culture according to methods developed in our laboratory (Goulet et al., 1996a). Briefly, the processing was performed within 2-3 hours following the bronchoscopy. Collagen being the major constituent of bronchi matrix, collagenase was chosen to digest the collagen matrix of the biopsies. The human bronchial biopsies were digested in $0.1 \%(0.2 \mathrm{U} / \mathrm{ml})$ collagenase $\mathrm{H}$ (Boehringer Mannheim, Montreal, Canada) prepared in Dulbecco-Vogt modification of Eagles medium (DMEM) culture medium containing $10 \mathrm{mM} \mathrm{CaCl}_{2}$ without any supplement. Tissues were digested overnight at $4^{\circ} \mathrm{C}$ because a collagenase digestion performed overnight at $37^{\circ} \mathrm{C}$ would reduce cell yield and viability. Homogenates were centrifuged for $10 \mathrm{~min}$ at $300 \mathrm{~g}$ and the cell pellets were resuspended in DMEM supplemented with $10 \%$ fetal calf serum (FCS).

All cells were plated in several 35-mm Petri dishes. The cultures were monitored daily and the dishes containing epithelial cells were selected for addition of some lethally irradiated 3T3 feeder cells (like it is done for skin epithelial cells, see Green et al., 1979). This technique allows the selection of pure epithelial cell populations (Goulet et al., 1996a). After 8-12 days in culture, bronchial epithelial cells had reached $85 \%$ confluency and were ready to be stored and subcultured. The bronchial epithelial cells were cultured according to the method established for human keratinocytes (Germain et al., 1993), originally described by Green et al. (1979), in a combination of DMEM with Ham's F12 in a 3:1 proportion (Gibco BRL, Burlington, Canada), supplemented with $24.3 \mu \mathrm{g} / \mathrm{ml}$ adenine (Sigma Chemicals, St-Louis, MO), $10 \mathrm{ng} / \mathrm{ml}$ human epidermal growth factor (EGF, Austral Biological, San Ramon, CA), $5 \mu \mathrm{g} / \mathrm{ml}$ bovine crystallized insulin, $5 \mu \mathrm{g} / \mathrm{ml}$ human transferrin (Boehringer Mannheim, Laval, Canada), 2x10-9 M 3,3',5', triiodo-L-thyronin (Sigma), $0.4 \mathrm{mg} / \mathrm{ml}$ hydrocortisone (Calbiochem, La Jolla, CA), 10-10 M cholera toxin (ICN Biochemicals, Montreal, Canada), 10\% reconstituted newborn calf serum (Immunocorp Sciences inc., Montreal, Canada) $100 \mathrm{IU} / \mathrm{ml}$ penicillin $\mathrm{G}$ and $25 \mu \mathrm{g} / \mathrm{ml}$ gentamicin (Sigma). Bronchial fibroblasts were obtained in the dishes which did not contain epithelial cells. They were cultured in DMEM supplemented with $10 \%$ fetal calf serum, $100 \mathrm{IU} / \mathrm{ml}$ penicillin $\mathrm{G}$ and $25 \mu \mathrm{g} / \mathrm{ml}$ gentamicin. They also reached $85 \%$ confluency after a week. The culture media was changed three times a week. All cultures were kept in an $8 \% \mathrm{CO}_{2}$ atmosphere at $37^{\circ} \mathrm{C}$.

\section{Human bronchial cell viability}

The viability of epithelial cells over 3-4 passages and of fibroblasts over 8-9 passages, were comparable between asthmatic and non-asthmatic cell populations (over $85 \%$ viability). However, the yields of epithelial cells obtained from asthmatic biopsies were about $60 \%$ lower in primary culture, compared to non-asthmatic biopsies. That is expected, since the epithelia of the asthmatic biopsies are often poorer in cells and sometimes disorganized. Moreover, considering that we isolated the cells from 6-10 microbiopsies (1-2 mm-diameter), we believe that our 
method can be considered as successful (Goulet et al., 1996a).

\section{Human bronchial cell markers}

The respective morphological features of each cell type are very different and were confirmed by specific immunolabeling. Epithelial cells were immunolabeled with anti-keratin antibodies and fibroblasts were labeled with antivimentin antibodies (Goulet et al., 1996a).

\section{Production of three-dimensional human bronchial equivalents (BEs)}

Step 1: Preparation of the mesenchymal layer of the BEs. The BEs were produced according to the method published by Auger et al. (1995), with a few modifications. Briefly, a mixture of bovine Type I collagen $(1.5 \mathrm{mg} / \mathrm{ml})$ was prepared by dissolving the powder overnight at $4^{\circ} \mathrm{C}$ in sterile $0.017 \mathrm{M}$ acetic acid. A solution of $0.84 \mathrm{ml}$ of DMEM 2.7X containing $200 \mathrm{IU} / \mathrm{ml}$ penicillin $\mathrm{G}$ and $50 \mu \mathrm{g} / \mathrm{ml}$ of gentamicin, $\mathrm{pH} 8.0$, was mixed with a second solution containing $0.56 \mathrm{ml}$ of FCS, $1.43 \mathrm{ml}$ of the stock collagen solution, $30 \mu \mathrm{l}$ of $\mathrm{NaOH} 0.7 \mathrm{~N}$ and $0.15 \mathrm{ml}$ of a HBFC suspension (1 $\mathrm{X} 106$ cells $/ \mathrm{ml})$. This mixture was quickly distributed in a bacteriological Petri dish (35-mm diameter) already containing the peripheral anchorage (sterile ring of Whatman paper), to produce the mesenchymal layers of each BE. The anchorage method, which prevents collagen lattice contraction by the cells, was used as described previously (L'Heureux et al., 1993; Auger et al., 1995). Non-asthmatic (na) and asthmatic (a) HBFC were seeded individually in different mesenchymal layers to produce naBE and aBE, respectively. The mesenchymal layers of the BE were covered with $2 \mathrm{ml}$ of DMEM supplemented with $10 \%$ FCS, $100 \mathrm{IU} / \mathrm{ml}$ penicillin $\mathrm{G}$ and $25 \mathrm{mg} / \mathrm{ml}$ gentamicin following collagen polymerization and cultured in this medium until their epithelialization.

Step 2: Epithelialization of the BE under submerged culture conditions. Four days later, the epithelialization was performed by seeding HBEC ( $8 \times 105$ cells / BE) on the mesenchymal equivalents, maintained under submerged culture conditions until a confluent epithelial cell layer was obtained. Again, non-asthmatic and asthmatic HBEC were seeded on the corresponding mesenchymal counterparts. In some groups of BEs, HBEC from non-asthmatic source were seeded on mesenchymal layers containing asthmatic HBFC and vice versa. During the first 3 days after epithelialization, all BEs were cultured in the medium used for the culture of HBEC (see the section Human bronchial cell isolation). On the fourth day after epithelialization, the BEs were cultured in serumfree medium, supplemented with $24.3 \mathrm{mg} / \mathrm{ml}$ adenine, $10 \mathrm{ng} /$ $\mathrm{ml}$ human EGF, $5 \mathrm{mg} / \mathrm{ml}$ bovine crystallized insulin, $5 \mathrm{mg} /$ $\mathrm{ml}$ human transferrin, $2 \times 10^{-9} \mathrm{M} 3,3^{\prime}, 5^{\prime}$, triiodo-L-thyronin, $0.4 \mathrm{mg} / \mathrm{ml}$ hydrocortisone, $10^{-10} \mathrm{M}$ cholera toxin, and $5 \times 10^{-8}$ $\mathrm{M}$ retinoic acid (RA). The culture media were changed daily.

Step 3: Culture at the air-liquid interface: The BEs were raised at the air-liquid interface as soon as a confluent layer of HBEC had covered their mesenchymal layers (6-8 days after epithelialization, depending on cell growth rates that could slightly vary from one experiment to another). The BEs were placed on Petri dishes containing an internal elevated support (Falcon No 3037). They were cultured at the airliquid interface for equal number of days (a period varying from 5 to 7 days, according to each experiment), in the same media used under submerged conditions, but in the absence of EGF and cholera toxin since these growth factors enhance the production of gelatinases by the cells, which degrade the collagen matrix of the mesenchymal layer in culture at the air-liquid interface (Auger et al., 1995). The culture media were changed daily. Each experiment was done 3 times on at least 3 samples per group of BEs tested.

\section{Conditioned medium}

The conditioned medium (CM) was obtained from confluent non-asthmatic HBFC populations. The cells were grown until they had reached $95 \%$ confluence in a 75 $\mathrm{cm}^{2}$ Falcon culture dish. During the conditioning period of $7 \mathrm{hrs}$, the HBFC were kept in $12 \mathrm{ml}$ of the same serum-free medium, used to culture the BEs under submerged conditions. Each sample of fresh CM was passed through a $0.22 \mu \mathrm{m}$ Millipore low-binding protein filter in order to eliminate all cellular fragments before being transferred on the various groups of BEs. Fresh CM was produced and used daily, without any dilution.

\section{Histological analysis}

All BEs were fixed with Bouin's solution and then embedded in paraffin. The $4 \mu \mathrm{m}$ thick sections were stained using two different methods: the hematoxylin, phloxine and saffron staining and the PAS staining. To eliminate cross-reaction with endogenous glycogen in situ, some $\mathrm{BE}$ sections were digested before PAS staining with a solution of $0.5 \%$ maltase (Fisher) in phosphate-buffered saline (PBS) for $30 \mathrm{~min}$ at $37^{\circ} \mathrm{C}$ and washed $10 \mathrm{~min}$ with distilled water. At least 10 tissue sections were analyzed for each BE tested. We observed the entire sections to take representative pictures.

\section{Electron microscopy}

Some BEs were also processed and mounted for transmission and scanning electron microscope analyses as previously described (Auger et al., 1993). Briefly, the samples were fixed with a solution of $2 \%$ glutaraldehyde in $0.1 \mathrm{M}$ sodium cacodylate buffer, $\mathrm{pH} 7.5$ for $24 \mathrm{hrs}$, postfixed with $1 \%$ osmium tetroxide, dehydrated with ethanol and coated with gold (Sputtercoater, Nanotech, Manchester, U.K.). Micrographs were made using Polaroid Polapan 400. A JEOL JSM-35CF scanning electron microscope was used.

\section{Gelatinase activity assay}

The active gelatinases secreted in the BE culture media were semi-quantitatively analyzed by zymography, according to the method described previously (Auger et al., 1995). Briefly, one sample of all BE culture supernatants were collected daily, filtered and immediately frozen at $70^{\circ} \mathrm{C}$ until use. At least 5 samples collected for each BE, during culture at the air-liquid interface, were analyzed by zymography. A constant volume $(25 \mu \mathrm{l})$ of each sample diluted in equal volume of $2 \mathrm{x}$ sample buffer was resolved under non-reducing conditions by SDS-PAGE (Laemmli, 1970). The gels were rinsed and equilibrated 

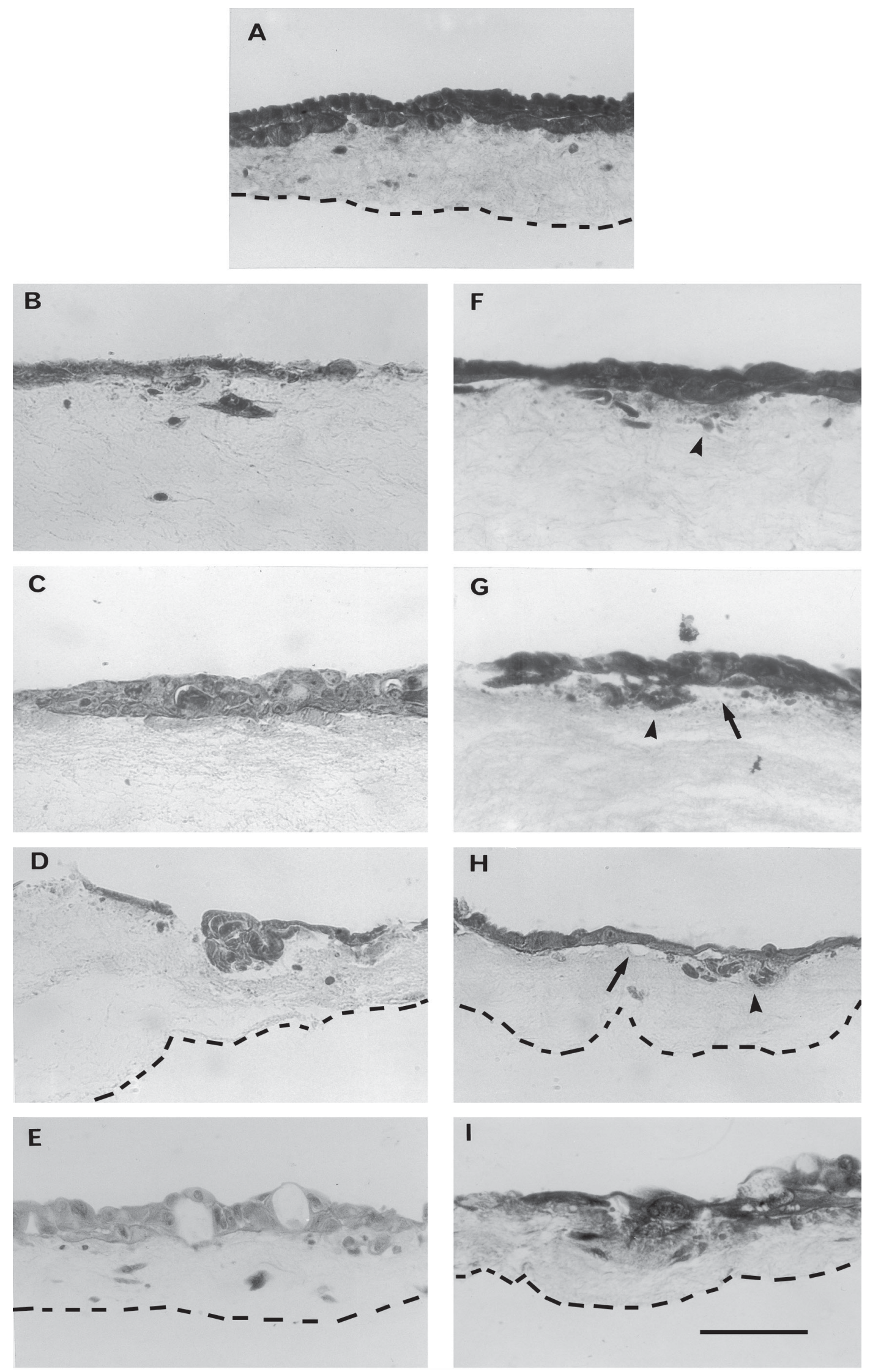

Figure 1. Histological analyses of different bilayered BE cultured for 6 days at the air-liquid interface in serum-free medium supplemented with RA. Masson trichrome staining of a BE produced with both non-asthmatic HBEC and HBFC (A), non-asthmatic HBEC and asthmatic HBFC (B-E), both asthmatic HBEC and HBFC (F-H), and asthmatic HBEC and non-asthmatic HBFC (I). The dashed line indicates the border of the mesenchymal layers in A, DE, H-I. Note the holes between the epithelial and the mesenchymal layers (arrows) and the cell debris under the basement membrane (arrowheads) of the BEs produced with asthmatic HBEC (F-I). Scale bar 36 $\mu \mathrm{m}$. 

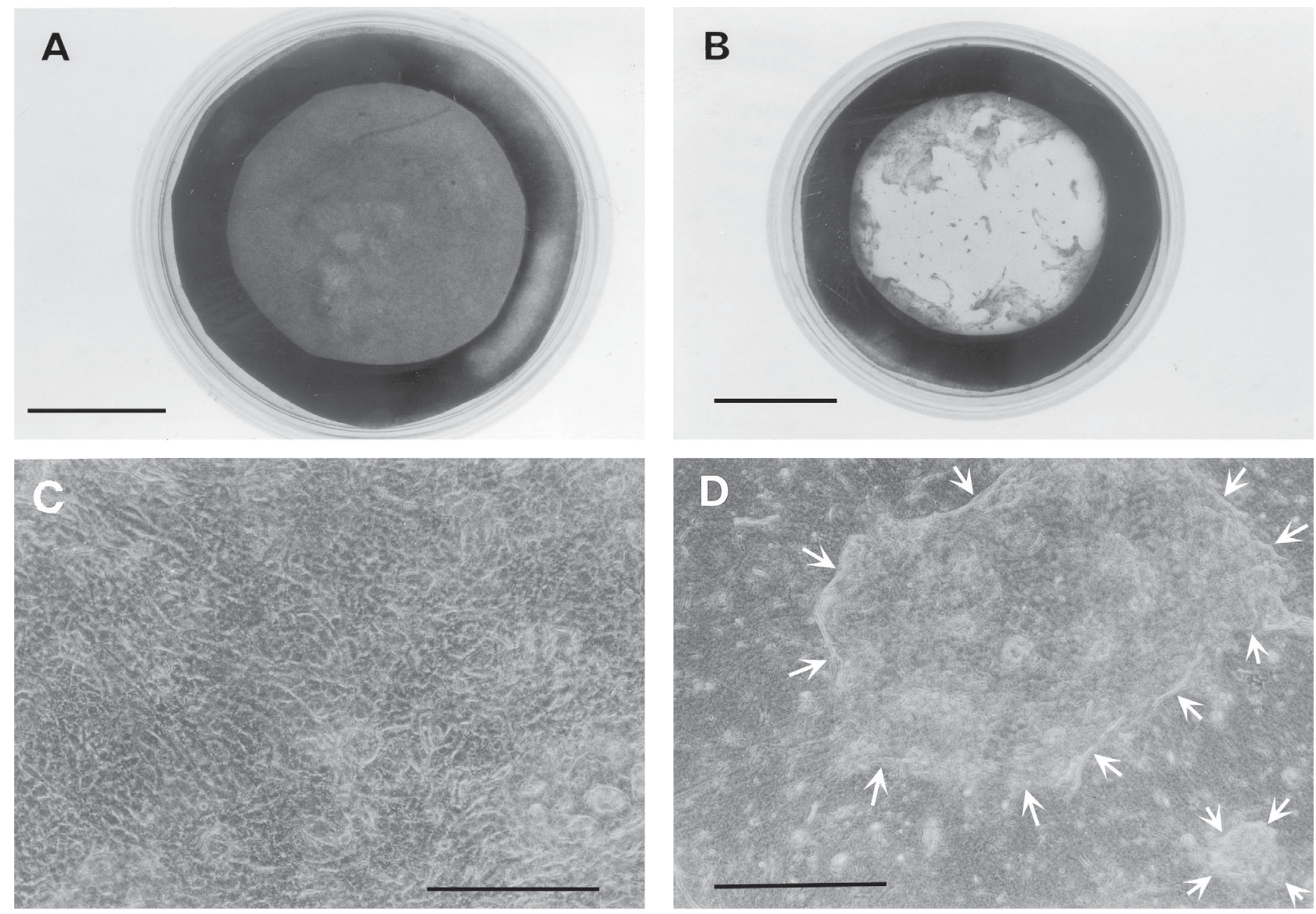

Figure 2. Macroscopic view of rhodanile red stained BEs (A and B). Non-asthmatic (A) and asthmatic (B) HBEC were grown on a BE mesenchymal counterpart containing non-asthmatic HBFC in serum-free medium supplemented with RA for 8 days under submerged culture conditions. Photomicrograph taken under phase constrast microscopy of BE sections after 18 days under the same culture conditions (C and D). Note the confluent layer of non-asthmatic HBEC (C), compared to the compact colonies (arrowheads indicate their borders) of asthmatic HBEC (D) slowly detaching from the mesenchymal counterpart. Scale bars: A-B, $0.5 \mathrm{~cm}$; C-D, $9 \mu \mathrm{m}$.

in the proper buffer systems and put overnight at $37^{\circ} \mathrm{C}$ in digestion buffer (50 mM Tris, $\mathrm{pH} 7.4$, containing $10 \mathrm{mM}$ $\mathrm{CaCl}_{2}$ and $100 \mathrm{mM} \mathrm{NaCl}$ ), under slow agitation. The gels were fixed with a $30 \%$ methanol solution containing $10 \%$ acetic acid and stained with $0.05 \%$ Coomassie blue prepared in the fixative. The gels were photographed and scanned.

\section{Results}

\section{Histological analysis of BEs}

Histological analyses were performed on 4 different groups of BEs cultured for 6 days at the air-liquid interface. One of the advantages of our bilayered $\mathrm{BE}$ is the possibility to produce different types of bioengineered tissues, containing HBEC and HBFC isolated from the same biopsies or from biopsies of different subjects (non-asthmatic or asthmatic). Thus, to evaluate and compare the growth of nonasthmatic and asthmatic HBEC on mesenchymal layers containing asthmatic and non-asthmatic HBFC, the four types of BEs were produced and analyzed.

The first group of BEs was produced using both nonasthmatic HBEC and HBFC, na/naBEs. Masson trichrome staining of a na/naBEs is shown in Figure 1A. The second group of BEs was produced by seeding non-asthmatic $\mathrm{HBEC}$ on a mesenchymal layer containing asthmatic HBFC, na/aBEs (Fig. 1, B-E). The third group of BEs was produced using both asthmatic HBEC and HBFC, a/aBEs (Fig. 1, F-H). In the fourth group of BEs, asthmatic HBEC were grown on a mesenchymal layer seeded with non-asthmatic HBFC, a/naBEs (Fig. 1I).

The thickness of the mesenchymal layers of the different BE groups showed differences upon culture time. As shown in figure 1, the mesenchymal layers of some a/aBEs (Fig. 1, F-G) and na/aBEs (Fig. 1, B-C) were degraded more slowly at the air-liquid interface, than the mesenchymal counterpart of na/naBEs (Fig. 1A), and depending on the asthmatic HBFC population seeded in the different groups of a/aBEs and na/aBEs.

The non-asthmatic HBEC reached a confluent state on the mesenchymal layers populated with non-asthmatic and asthmatic fibroblasts: na/naBEs (Fig. 1A) and na/aBEs (Fig. 1, B-E), respectively. However, these cells covered the mesenchymal layer of aBEs one day later than those seeded in naBEs (data not shown). Similarly, HBEC isolated from asthmatic subjects grew more slowly (in 8 days under submerged culture conditions) on all mesenchymal layers, 

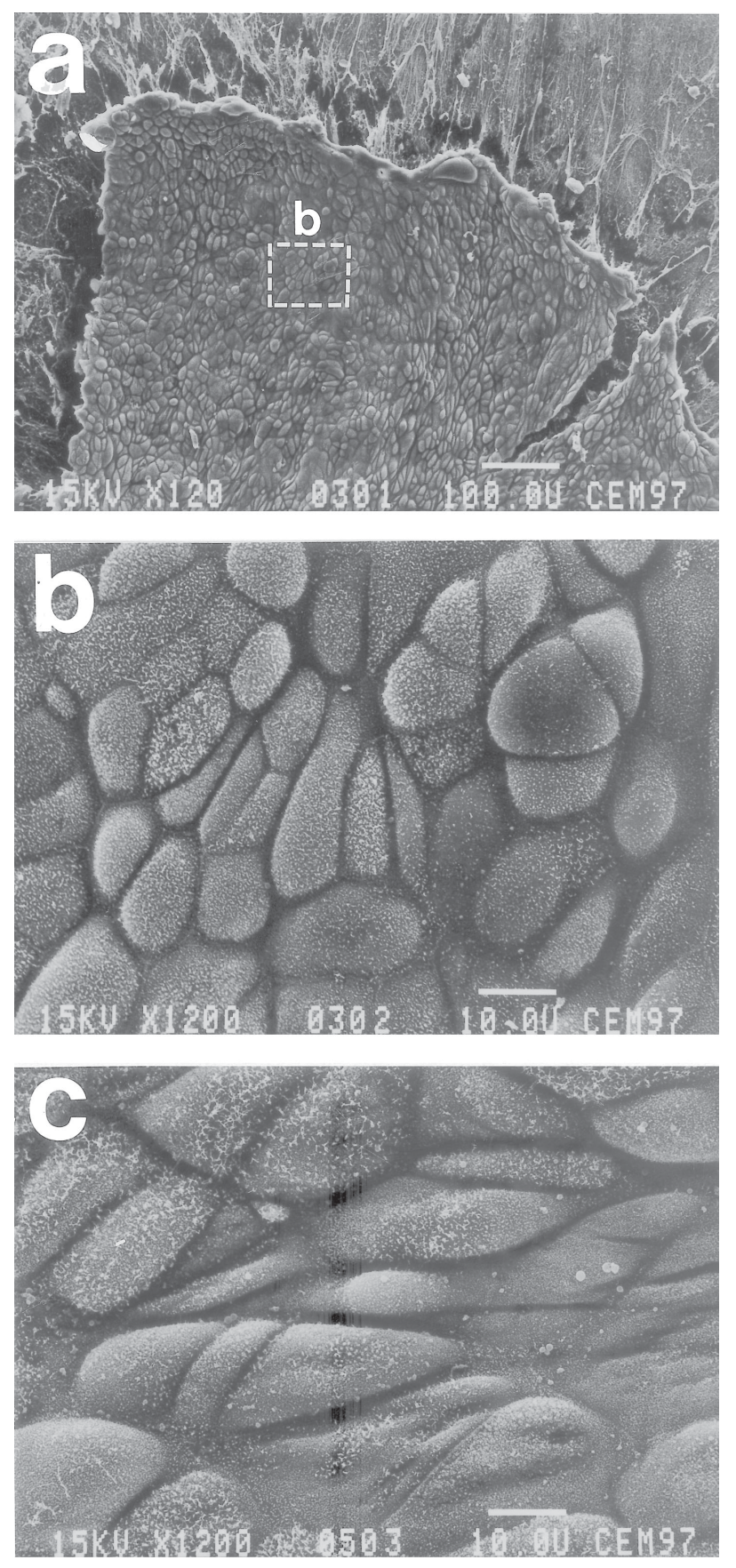

Figure 3. Scanning electron microscopy analyses of ciliated HBEC in two different BE cultured for 7 days at the air-liquid interface. Photomicrograph of a small patch of asthmatic HBEC (a) remaining after the partial detachment of the epithelial layers on a BE mesenchymal layer seeded with asthmatic HBFC, shown at higher magnification (b) and compared to non-asthmatic HBEC (c) grown on a mesenchymal counterpart seeded with non-asthmatic HBFC.

populated with asthmatic HBFC (Fig, 1, F-H) and non-asthmatic HBFC (fig 1I). However, after reaching a confluent state on the various mesenchymal counterparts, the asthmatic HBEC slowly started detaching in plates from the matrix, one or two days later. Some holes remained in the reconstructed epithelia thereafter, combined to the presence of cell debris observed under the basement membrane of all a/aBEs and a/naBEs (Fig. 1, F-I). Compared to the main-
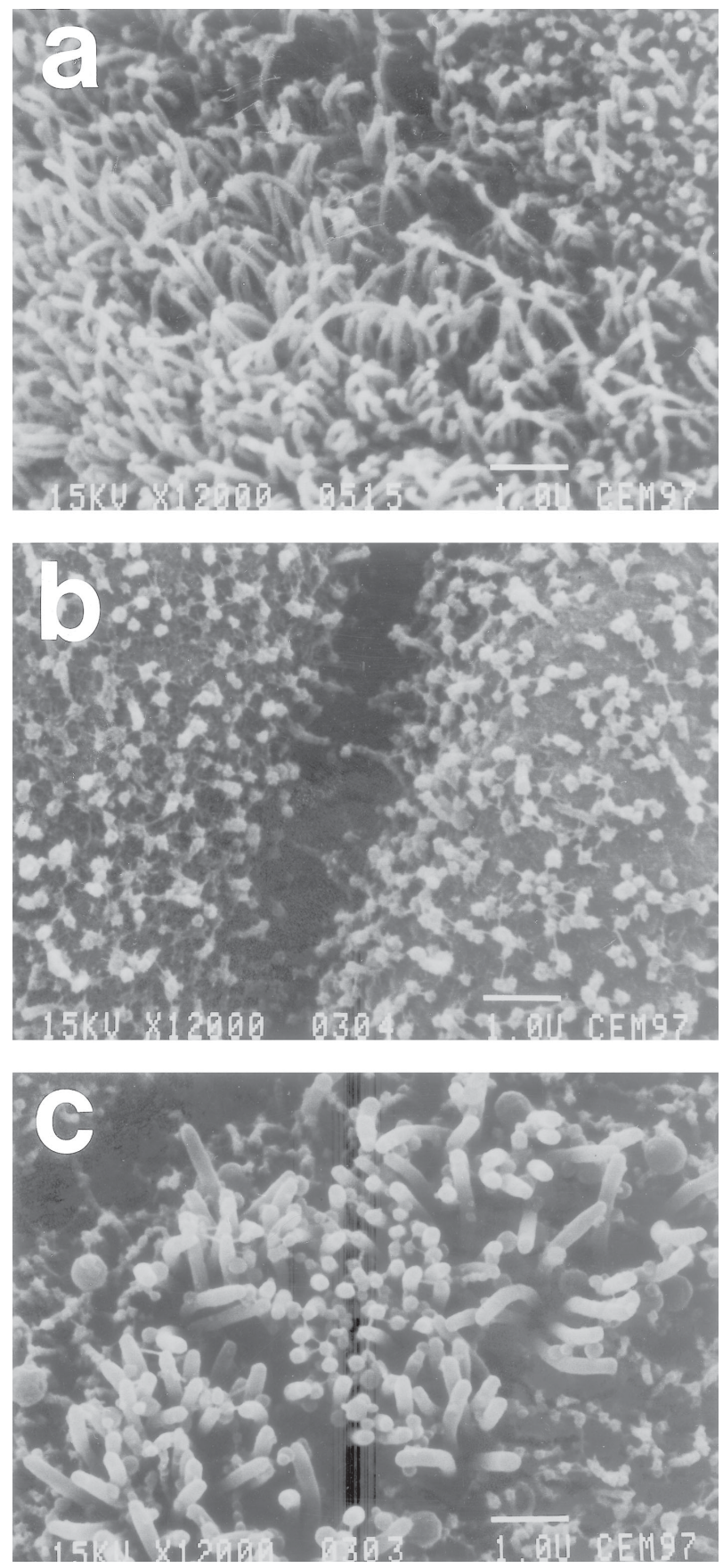

Figure 4. Scanning electron microscopy analyses of ciliated HBEC in two different BE cultured for 7 days at the air-liquid interface. Photomicrograph of a non-asthmatic HBEC (a) grown on a mesenchymal counterpart seeded with non-asthmatic HBFC. Note the numerous and long cilia on its apical pole (a). Photomicrographs of asthmatic HBEC on a BE mesenchymal layer seeded with asthmatic HBFC (b-c). Note the differences in the number and length of cilia among different asthmatic cells at the same magnification.

tenance of a confluent epithelial layer of non-asthmatic HBEC on all BEs (Fig. 2A), the striking detachment of the asthmatic HBEC could be seen macroscopically after rhodanile red staining in the corresponding BEs (Fig. 2B). These observations were confirmed under phase constrast microscopy of BE sections, (Fig. 2, C-D). Indeed, the confluent layer of non-asthmatic HBEC (Fig. 2C), was very different from the compact colonies of asthmatic HBEC 
(Fig. 2D), that were slowly detaching from their mesenchymal counterpart.

\section{Electron microscopy analyses of the various groups of BEs}

Scanning electron microscopy analyses confirmed the partial detachment of the asthmatic HBEC from the mesenchymal layers of the various BEs (Fig. 3a). A small patch of asthmatic HBEC contained ciliated asthmatic HBEC in a/aBEs cultured for 7 days at the air-liquid interface (Fig. $3 a-b)$. Similar observations were made on a/naBEs (data not shown). All non-asthmatic HBEC grown in a naBE under the same culture conditions showed a ciliated layer of cells of more elongated morphology (Fig. 3c). Similar observations were also made in na/aBEs (data not shown). Transmission electron microscopy analyses showed collagen fibers surrounded by bronchial fibroblasts in the mesenchymal layers of the various groups of BEs, but no difference was detected between their matrix ultrastructural features (data not shown).

\section{Ciliogenesis in BEs}

Ciliogenesis of the HBEC isolated from non-asthmatic and asthmatic tissues and grown within the different groups of BEs, was assessed by scanning electron microscopy analyses, after 7 days at the air-liquid interface. Ciliogenesis occurred in all HBEC, independently from their tissue of origin. However, longer and more numerous cilia were observed on the apical pole of all non-asthmatic HBEC (Fig. 4a). In contrast, sparsely distributed shorter cilia (Fig. $4 b)$ were observed on some HBEC isolated from asthmatic tissues, while in some others of the same population, ciliogenesis was comparable to non-asthmatic cells (Fig. $4 c)$. Thus, ciliogenesis occurred at various stages in the asthmatic HBEC but was higher and more consistently observed in non-asthmatic HBEC, independently of the mesenchymal layers the cells were seeded on.

\section{Conditioned medium}

On the basis of several publications reporting the important and beneficial effects of epithelium-mesenchyme interactions through factors secreted and exchanged between both types of tissues (Bouvard et al., 1992; Goulet et al., 1996b; Paquette et al., 2003), the effects of non-asthmatic $\mathrm{HBFC}$ was assessed on the growth of non-asthmatic HBEC in na/naBEs and na/aBEs. The growth of the non-asthmatic HBEC in the different BEs, cultured for 7 days under submerged conditions, was assessed by rhodanile red staining. Non-asthmatic HBEC grown on a naBE were used as the positive control corresponding to the highest epithelial cell density observed in absence of CM (Fig. 5, picture 1). Nonasthmatic HBEC were also grown on two types of mesenchymes containing different asthmatic populations of HBFC (Fig. 5, pictures 2-3). As expected, the addition of $\mathrm{CM}$ on na/naBEs did not improve the growth of the HBEC (data not shown). However, non-asthmatic HBEC, seeded on mesenchymal layers populated with asthmatic HBFC (na/aBEs) and cultured in CM, grew much faster (Fig. 5, pictures 2-3, CM) than the corresponding BEs, cultured in the same medium but not conditioned by the non-asthmatic HBFC (Fig. 5, pictures 2-3).

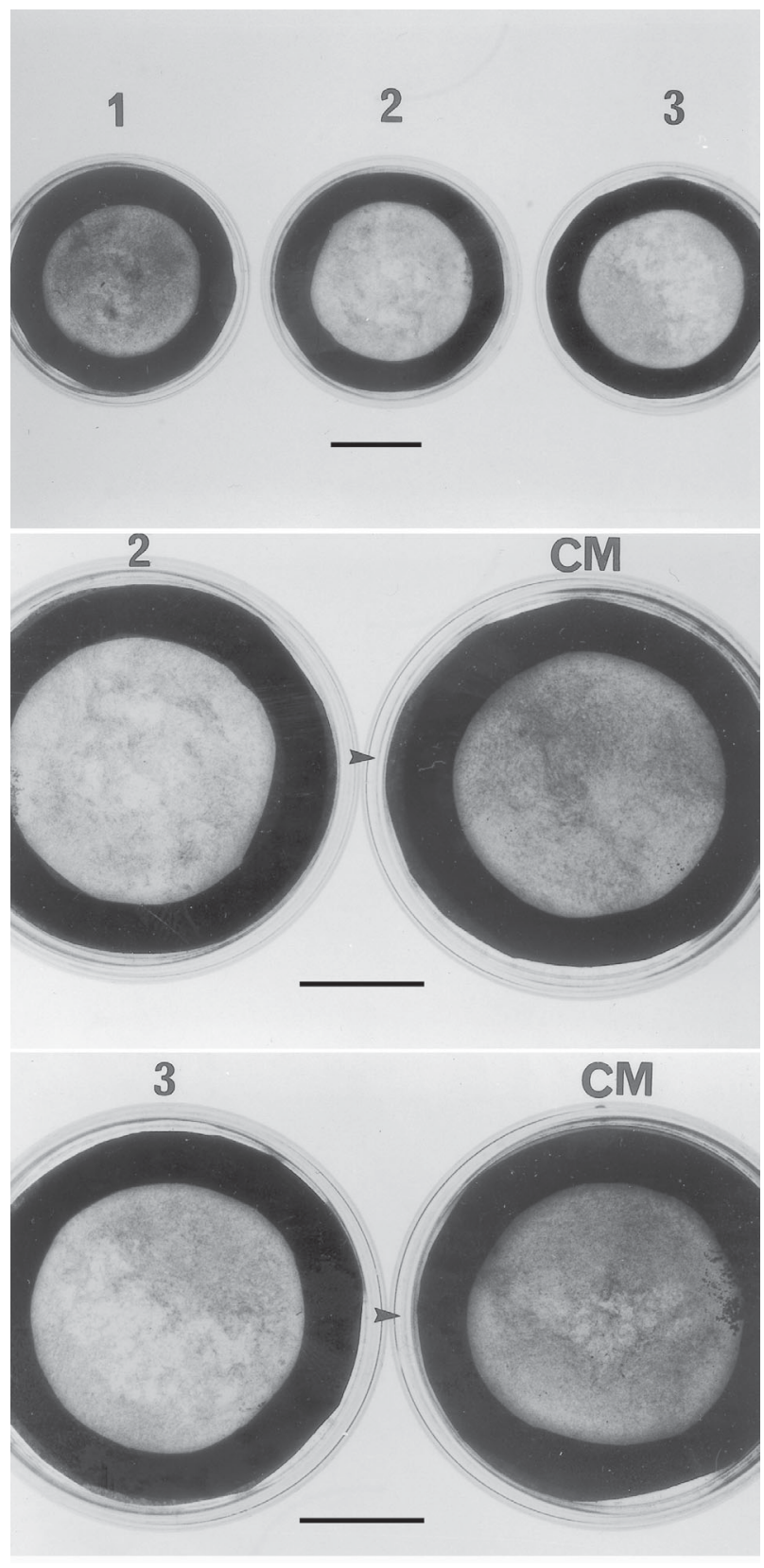

Figure 5. Macroscopic view of rhodanile red stained BE. Non-asthmatic HBEC were grown on a BE mesenchymal counterpart containing non-asthmatic HBFC (1) and two different asthmatic populations of HBFC (2$3)$, cultured in serum-free medium supplemented with RA (2-3) and conditioned medium (CM) for 7 days under submerged conditions. Note the differences in the density of non-asthmatic HBEC observed in response to the HBFC isolated from non-asthmatic (1) and asthmatic (2-3) tissues, seeded in the mesenchymal counterparts of the different BE. Note the stimulatory effects of the addition of CM (conditioned by non-asthmatic HBFC) on the growth of HBEC seeded on the two mesenchymal layers containing the respective asthmatic $\mathrm{HBFC}$ used to produce the BE 2 and 3. Scale bars $0.5 \mathrm{~cm}$. 


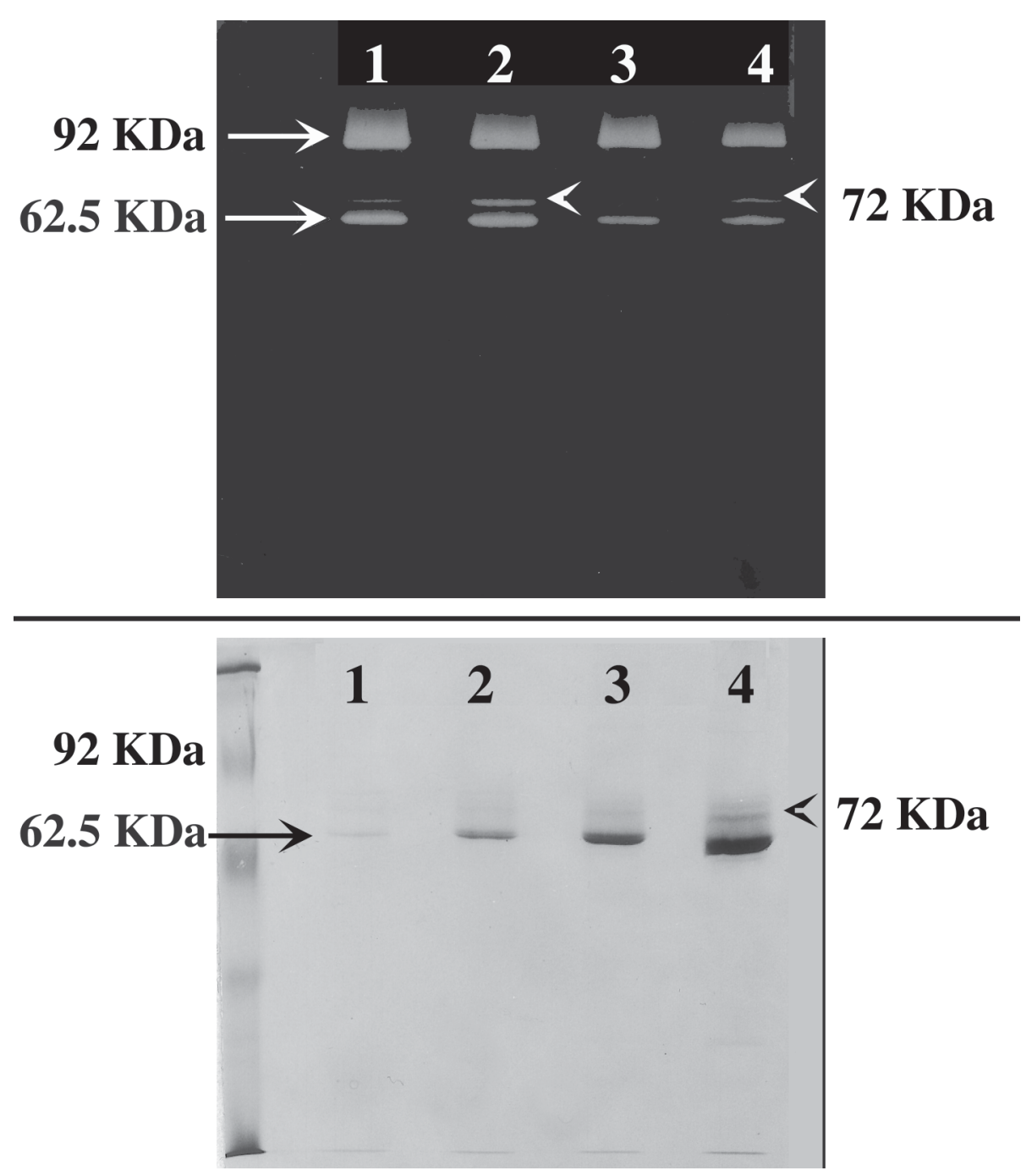

Figure 6. Zymograms (top) and corresponding SDS-PAGE (bottom) showing the relative gelatinase activities analyzed from supernatants of naBE (lane 1), of two different a/aBEs (lanes 2 and 3) and of an a/aBE cultured in the medium conditioned by HBFC (lane 4). The arrowheads indicate the gelatinase A (MMP-2, $72 \mathrm{kDa})$. The arrows indicate the gelatinase $\mathrm{B}(\mathrm{MMP}-9,92 \mathrm{Kda})$ and the active form of gelatinase $\mathrm{A}(62.5 \mathrm{kDa})$.

\section{Gelatinases secretion by human bronchial cells}

Gelatinases A, zymogen of MMP-2 (proMMP-2, $72 \mathrm{kDa}$ ), and activated MMP-2 (active MMP-2, $62.5 \mathrm{kDa}$ ) and $\mathrm{B}$ (MMP-9, $92 \mathrm{kDa}$ ) were recently described as secretion products of HBFC and HBEC, respectively (Hoshino et al., 1998). The gelatinases secreted by the BEs cultured at the air-liquid interface were analyzed by zymography. At least 5 different samples were investigated for each BE tested and we show in Fig. 6 a representative zymogram. Our results showed that the active form of gelatinase A $(62.5 \mathrm{kDa})$ was secreted by all HBFC in the BE supernatants (Fig. 6, lanes 1-4), including the supernatant of a/aBEs cultured in presence of CM (Fig.6, lane 4). The gelatinase B (92 kDa) was also actively secreted by the various HBEC in all BE supernatants (Fig, 6, lanes 1-4), but the zymograms showed slightly lower activities of this enzyme in the supernatants taken from a/aBEs (Fig. 6, lane 2-4), compared to na/naBEs (Fig. 6, lane 1).

Interestingly, the sample that contained the lowest amounts of gelatinase $\mathrm{A}(72 \mathrm{kDa})$, associated with fibroblast secretion products, corresponded to the culture supernatant resolved in lane 3 (Fig. 6), collected from the a/aBE. This $\mathrm{BE}$ showed the thicker mesenchymal layer on histological sections (Fig. 1F), compared to the other BEs cultured for 6 days at the air-liquid interface. This sample (Fig. 6, lane 3 ), was compared to the supernatant of another a/aBE, produced with the same cells, but cultured in presence of medium conditioned by non-asthmatic HBFC (Fig. 6, lane 4). The CM-stimulated-a/aBE (Fig. 6, lane 4), contained more of the precursor form of the gelatinase $\mathrm{A}(72 \mathrm{kDa})$. It also contained less of the active $62.5 \mathrm{kDa}$ gelatinase than the other BEs (Fig. 6, lanes 1-3). However, this a/aBE showed a slightly thinner mesenchymal layer on histological analyses than the na/naBEs (Fig. 1A). Thus, it is difficult to establish a correlation between the amounts of gelatinases secreted in the culture supernatants of the various BEs and the rate of degradation of their mesenchymal layers in culture. Other types of proteases may be involved in this process. 


\section{Discussion}

The production of bioengineered BEs with non-asthmatic and asthmatic cells allowed the comparative analyses of severa1 histologic and functional parameters in vitro. Our results suggest that HBEC isolated from asthmatic subjects can grow on a mesenchymal layer seeded with nonasthmatic and asthmatic HBFC. However, if they grow better on a non-asthmatic than on an asthmatic type of mesenchymal layer, they all started to detach from their mesenchymal support one to two days after reaching confluence. Such process continued thereafter, under submerged culture conditions and subsequently, at the air-liquid interface. In contrast, the non-asthmatic HBEC never detached from any type of mesenchymal layer tested. When asthmatic HBEC are grown in monolayers on plastic, they can be maintained in culture for at least 3-4 passages without detaching from the culture dishes, in a medium supplemented with serum. When grown in BEs, their detachment from the various mesenchymal layers, correlates with the formation of holes and the deposition of cell debris at the interface epithelium-mesenchyme. These data suggest that asthmatic HBEC fail to maintain stable points of anchorage with the matrix support, probably due to an absence or a loss of membranous anchorage proteins or receptors. In absence of serum that contains several growth factors and fibronectin, such defect may be observed earlier. We may believe that the behavior of the HBEC isolated from asthmatic subjects seeded on BEs is comparable to the observations reported in asthmatic tissues in vivo when desquamation occurs (Jeffery et al., 1989).

Ciliogenesis was observed, at least partially, in all groups of BEs. However, several asthmatic HBEC did not show cilia as numerous and/or as long as the non-asthmatic HBEC. These data suggest that the asthmatic HBEC do not reach the same level of differentiation within a given population isolated from the same tissues. Such observations were also reported from histologic analyses of severe asthmatic bronchi, whereas several non-ciliated cells were detected at the surface of their epithelium (Jeffery et al., 1989). Again, it was not surprising to observe this phenomena in our $\mathrm{BE}$ in vitro.

The non-asthmatic HBEC grew more slowly within a $\mathrm{na} / \mathrm{aBE}$ and the histologic organization of the na/aBEs was less compact than in the na/naBEs. Inversely, the asthmatic HBEC grew faster on a mesenchymal layer containing nonasthmatic HBFC than in a aBE. These data strongly suggest that the HBEC respond to some factors secreted by the HBFC up to a certain degree, and may as well release other factors to the mesenchymal cells. It was previously demonstrated in vitro that epithelial cells are an important regulator of airway remodeling by means of paracrine control of bronchial fibroblasts (Zhang et al., 1999). Other evidence supporting the existence of epithelium-mesenchyme interactions in the BEs is the positive effect of the CM collected from non-asthmatic HBFC on the growth of nonasthmatic HBEC on a mesenchymal layer seeded with asthmatic HBFC. It seems that the CM contains cytokines that could compensate a lack of factors secreted by the asthmatic HBFC in the na/aBE.
The comparative analyses of the active gelatinases secreted in the BEs culture supernatants showed some differences, but the $92 \mathrm{kDa}$ and the $62.5 \mathrm{kDa}$ gelatinases were detected in all BE supernatants. The supernatant of the a/ aBE showing the thicker mesenchymal layer may be explained by a lower secretion of gelatinase $\mathrm{A}(72 \mathrm{kDa})$ or by the synthesis of other collagen types, such as Type $\mathrm{V}$ (Hoshino et al., 1998). The apparent sub-epithelial fibrosis associated with asthma is postulated to be caused by fibroblasts. These cells are involved in collagen synthesis and remodelling and their functional status may be modulated by cytokines secreted by inflammatory or epithelial cells (Brewster et al., 1990; Roche, 1991; Gauldie et al., 1992). Thus, several hypotheses could be considered to explain our results. The zymograms on gelatin, a substrate rich in collagen Type I, do not reveal several other collagenases and proteases that may be secreted in different amounts by asthmatic and non-asthmatic cells present in our different BEs. It would be interesting to assess the effects of specific inhibitors of MMP-9 and MMP-2 on the maintenance of the mesenchymal layers of the different groups of BEs.

Taken together, we conclude that the tissue-engineered human BE is a good model to investigate cellular mechanisms involved in bronchial disorders like asthma. This approach allows the analyses of several parameters such as the bronchial cell growth and differentiation, their organization and their secretion products in vitro. Comparative toxicological and pharmacological studies of various human bronchial cell populations can be achieved in vitro, using $\mathrm{BE}$ as a precious alternative to animal use. Eventually, other cell types, such as immune or smooth muscle cells could be added to the model to investigate more complex interactions.

\section{Acknowledgements}

The authors are grateful to Dr Hilda López Valle, M.D., pathologist, for her critical analyses of the histological and ultrastructural properties of the bronchial equivalents, $\mathrm{Mr}$. Aristide Pusterla for his contribution in electron microscopy analyses, Mr. Claude Marin for photographic assistance, Mrs Nathalie Tremblay for her technical assistance on this project. Mr. Jean-Sébastien Paquette was recipient from the NSERC fellowship and a honorary fellowship from Laval University. Drs F. Goulet, F.A. Auger, and L.-P. Boulet were recipients of Scolarships from the Fonds de la Recherche en Santé du Québec.

\section{References}

American Thoracic Society (1987) Standards for the diagnosis and care of patients with chronic obstructive pulmonary disease (COPD) and asthma. Am Rev Respir Dis 136: 225-244.

Auger FA, Guignard R, Lopez Valle CA, Germain L (1993) Role and inocuity of Tisseel $\AA$, a tissue glue, in the grafting process and in vivo evolution of human cultured 
epidermis. Br J Plastic Surg 46: 136-142.29.

Auger FA, Lopez Valle CA, Guignard R, Tremblay N, Noël B, Goulet F, Germain L (1995). Skin equivalents produced using human collagens. In Vitro Cell Dev Biol 31: 432-439.

Baeza-Squiban A, Boisvieux-Ulrich E, Guilianelli C, Houcine O, Geraud G, Guennou C, Marano F (1994) Extracellular matrix-dependent differentiation of rabbit tracheal epithelial cells in primary culture. In Vitro Cell Dev Biol Anim 30A: 56-67.

Bellows CG, Melcher AH, Aubin JE (1982) Association between tension and orientation of periodontal ligament fibroblasts and exogenous collagen fibres in collagen gels in vitro. J Cell Sci 58: 125-138.

Boulet LP, Turcotte A, Boutet M, Milot J, Côté J, Malo JL, Cartier A, Dugas M, Laviolette M (1993) Correlations between airway inflammation and responsiveness to metacholine in subjects with asthma, rhinitis, reactive airways dysfunction syndrome (RADS), chronic laugh and normals. Eur Respir J 6(S17): 265S (abstract).

Bouvard V, Germain L, Rompré P, Roy B, Auger FA (1992). Influence of dermal equivalent maturation on a skin equivalent development. Biochem Cell Biol 70: 34-42.

Brewster CEP, Howarth PH, Djukanovic R, Wilson J, Holgate ST, Roche WR (1990) Myofibroblasts and subepithelial fibrosis in bronchial asthma. Am J Respir Cell Mol Biol 3: 507-511.

Chakir J, Page N, Hamid Q, Laviolette M, Boulet LP, Rouabhia M (2001) Bronchial mucosa produced by tissue engineering: a new tool to study cellular interactions in asthma. J Allergy Clin Immunol 107: 36-40.

Chung KF (1996) Effects of nedocromil sodium on airway neurogenic mechanisms. J Allergy Clin Immunol 98: S112-S117.

Davenport EA, Nettesheim P (1996) Regulation of mucociliary differentiation of rat tracheal epithelial cells by type 1 collagen gel substratum. Am J Respir Cell Mol Biol 14: 19-26.

De Jong PM, Van Sterkenburg MAJA, Hesseling SC, Kempenaar JA, Mulder AA, Mommaas AM, Dijkman JH, Ponec M (1994) Ciliogenesis in human bronchial epithelial cells cultured at the air-liquid interface. Am J Respir Cell Mol Biol 10: 271-277.

DiCosmo B, Goba G, Picarella D, Elias JA, Rankin JA, Stripp B, Whitset JA, Flavell RA (1995). Expression of interleukin- 6 by airway epithelial cells. Effects on airway inflammation and hyperreactivity in transgenic mice. Chest 107: 131S (abstract).

Djukanovic R, Roche WR, Wilson JW, Beasley CRW, Twentyman OP, Howarth PH, Holgate ST (1990) Mucosal inflammation in asthma. Am Rev Respir Dis 142: 434-457.

Gauldie J, Jordana N, Cox G, Ohtoshi T, Dolovich J, Denburg J (1992) Fibroblasts and other structural cells in airway inflammation. Am Rev Respir Dis 145: S14-S17.

Germain L, Rouabhia M, Guignard R, Carrier L, Bouvard V, Auger FA (1993) Improvement of human keratinocyte isolation and culture using thermolysin. Burns 19: 99-104.

Goulet F, Boulet LP, Chakir J, Tremblay N, Dubé J, Laviolette M, Auger FA (1996a). Morphological and functional properties of bronchial cells isolated from normal and asthmatic subjects. Am J Resp Cell Mol Biol 15, 312318.

Goulet F, Poitras A, Rouabhia M, Cusson D, Germain L, Auger FA (1996b) Stimulation of human keratinocyte proliferation through growth factor exchanges with dermal fibroblasts in vitro. Burns 22: 107-112.

Goulet F, Germain L, Rancourt D, Caron C, Normand A, Auger FA (2000) Tendons and ligaments. In: Principles of Tissue Engineering, 2nd ed. Lanza R, Langer R, Vacanti J (eds). Academic Press, San Diego, pp 711-722.

Gray EG, Guzman K, Davis CW, Abdullah LH, Nettesheim P (1996) Mucoci1iary differentiation of serially passaged normal human tracheobronchial epithelial cells. Am J Respir Cell Mol Biol 14: 104-112.

Green H, Kehinde O, Thomas J (1979) Growth of cultured human epidermal cells into multiple epithelia suitable for grafting. Proc Natl Acad Sci (USA) 76: 5665-5668.

Hoshino M, Nakamura Y, Sim J, Shimojo J, Isogai S (1998) Bronchial subepithelial fibrosis and expression of matrix metalloproteinase-9 in asthmatic airway inflammation. J Allergy Clin Immunol 102: 783-788.

Jeffery PK, Wardlaw AJ, Nelson FC, Collins JV, Kay AB (1989) Bronchial biopsies in asthma. Am Rev Respir Dis 140: 1745-1753.

Juniper EF, Cockcroft DW, Hargreave FE (1991) Tidal breathing method. In Histamine and Methacholine Inhalation Tests: Laboratory Procedure and Standardization. Canadian Thoracic Society. AB Draco, Lund, Sweden.

Laemmli UK (1970) Cleavage of structural proteins during the assembly of the head of bacteriophage T4. Nature 227: 680-685.

Langer R, Vacanti JP (1993) Tissue engineering. Science 260: 920-926.

L'Heureux N, Germain L, Labbé R, Auger FA (1993) In vitro construction of a human blood vessel from cultured vascular cells. J Vasc Surg 17: 499-509.

Lopez Valle CA, Auger FA, Rompré P, Bouvard V, Germain L (1992) Peripheral anchorage of dermal equivalents. Br J Dermatol 127: 365-371.

Opazo-Saez A, Pare PD (1994) Stimulus-response relationships for isotonic shortening and isometric tension generation in rabbit trachealis. J Appl Physiol 77: 16381643.

Paquette JS, Tremblay P, Bernier V, Auger FA, Laviolette M, Germain L, Boutet M, Boulet LP, Goulet F (2003) Production of tissue-engineered three-dimensional human bronchial models. In Vitro Cell Dev Biol-Animal 39: 213220.

Roche WR (1991) Fibroblast and asthma. Clin Exp Allergy 21: 545-548.

Shichinoke K, Shimizu M, Kurokawa K (1996) Effect of M-711 on experimental asthma in rats. J Vet Med Sci 58: $55-59$.

Widdicombe J (1996) The tracheobronchial vasculature: a possible role in asthma. Microcirculation 3: 129-141.

Zhang S, Smartt H, Holgate ST, Roche WR (1999) Growth factors secreted by bronchial epithelial cells control myofibroblast proliferation: an in vitro co-culture model of airway remodeling in asthma. Lab Invest 79: 395405 . 


\section{Discussion with Reviewers}

K. Qvortrup: The authors report a morphological difference between controls and cells from asthmatics. Is it possible to give more details (e.g., \% of elongated cells or difference between cell length/width)?

Authors: We did not measure the morphological cell features. We noticed some morphological differences which were observed after the cells were seeded in the three-dimensional reconstructed tissues and we wanted to bring it to the reader's attention. This work would be interesting to do but we did no think that this information was essential at this point.

K. Qvortrup: It might be useful to address in the discussion the possible limitations of the method: the immune system is expected to play a role in the development of allergic diseases. Isn't the absence of immune cells in the system a problem? Would it be useful to supplement the culture medium with patient serum?

Authors: The reviewer is right but we must consider several other issues, if we want to introduce immune cells in the model. We present in this manuscript a model to study epithelium-mesenchyme cell interactions in asthma. If we wanted to add some immune cells in the system, we could supplement the culture medium with patient serum, containing monocytes, polymorphonuclear cells, etc. However, would it insure that the key immune cells, involved in asthmatic reactions, would be present and viable in the model? Moreover, would these cells retain their capacity to interact with each other in the model? In vivo, many interactions occur between the various immune cells themselves and between the immune cells and the epithelial or mesenchymal cells. These interactions are complex and not well understood. We chose to develop a simplified model to assess if the mesenchymal and epithelial cells, isolated from asthmatic tissues, could retain some of their properties in a reconstructed bronchial tissue. A second step of the project could be to investigate the effect of some known immune cell cytokines, such as interleukins, on normal and asthmatic bronchial constructs. Several studies could also be performed to assess the effects of anti-inflammatory molecules, such as receptor inhibitors, using the bronchial models. Such experiments have to be well planned and performed under controlled culture conditions. We established the model to investigate these questions. We plan to add a smooth muscle cell layer to the model as well, since these cells probably respond to immune cell signals as well.

P. Bongrand: Supposedly some specimens were processed and mounted for transmission electron microscopy. Such data is not presented. This would have been very useful, particularly illustrations of the detachment zones and subepithelial debris in BE with aHBEC.

Authors: The best results obtained from transmission electron microscopy showed the good alignment of the fibroblasts and the surrounding collagen fibers in the mesenchymal layers. The pictures were not included in the paper because they did not show anything really new. We did not get good pictures of the epithelia by transmission electron microscopy. We obtained original and clear pictures of the epithelia by scanning electron microscopy and this is why we chose to add them in the article. We agree with the reviewer that it could have been interesting to see the detachment zones but we focused on the ciliated cells.

P. Bongrand: Ciliogenesis: cilia on "apical pole" of a nonasthmatic HBEC are displayed. What did the rest of the cell surface display?"

Authors: We observed the epithelial layers on tissue sections stained with Masson trichrome under phase contrast microscopy. We repeatedly saw mononucleated epithelial cells with cilia restricted to their apical pole, arranged tightly besides each other to form a pseudostratified layer. No cilia were observed at the intercellular junctions. Sometimes, a goblet cell (labeled with PAS, a mucoglycoprotein-staining method) would be present between two ciliated cells (Figure 1E). We recently published a paper on non-asthmatic HBEC, which includes pictures of a group of ciliated cells analyzed by scanning electron microscopy (Paquette et al., 2003, text reference). 